\title{
Efficacy and Safety of Direct Oral Anticoagulant for Treatment of Atrial Fibrillation in Cerebral Amyloid Angiopathy
}

\author{
Koichi Narita ${ }^{1}$, Eisuke Amiya ${ }^{1,2}$, Issei Komuro ${ }^{1}$ \\ 1. Department of Cardiovascular Medicine, The University of Tokyo, Tokyo, JPN 2. Department of Therapeutic Strategy \\ for Heart Failure, The University of Tokyo, Tokyo, JPN
}

Corresponding author: Eisuke Amiya, amiyae-tky@umin.ac.jp

\begin{abstract}
A 75-year-old man with a history of atrial fibrillation (AF) and anticoagulant therapy presented with a headache. Cerebral amyloid angiopathy (CAA) was diagnosed after MRI of the brain revealed cortical superficial siderosis, lobar intracerebral hemorrhage, and lobar microbleeds. Anticoagulant therapy was carefully discontinued. Several years later, he was admitted with sudden onset left upper-extremity weakness. In addition to CAA bleeding lesions, a diffusion-weighted brain MRI showed multiple infarct lesions of high signal intensity. The administration of edoxaban $7.5 \mathrm{mg} /$ day (later increased up to $30 \mathrm{mg} / \mathrm{day}$ ) prevented ischemic stroke recurrence without exacerbation of cerebral bleeding. This could indicate that CAA patients with $\mathrm{AF}$ who had previous adverse effects from warfarin can safely use newer direct oral anticoagulants, such as edoxaban, to prevent ischemic stroke without danger of cerebral hemorrhage. The superiority of edoxaban as compared with warfarin might be due to its antioxidant effect because vascular oxidative stress plays a causal role in CAA-induced cerebrovascular dysfunction, CAA-induced cerebral hemorrhage, and CAA formation itself. We explained the beneficial effect of edoxaban for CAA by the mechanism of oxidative stress in the paper.
\end{abstract}

Received 08/17/2020

Review began 08/17/2020 Review ended 08/25/2020 Published 08/30/2020

() Copyright 2020 Narita et al. This is an open access article distributed under the terms of the Creative Commons Attribution License CC-BY 4.0., which permits unrestricted use, distribution, and reproduction in any medium, provided the original author and source are credited.
Categories: Cardiology, Internal Medicine, Neurology

Keywords: cerebral amyloid angiopathy, atrial fibrillation, anticoagulant therapy, direct oral anticoagulant, oxidative stress

\section{Introduction}

Atrial fibrillation (AF) is increasing in prevalence with aging, and the main cause of cerebral infarction is AF. The anticoagulant therapy is effective in reducing the risk of cerebrovascular infarction in $\mathrm{AF}$ [1,2]; however, there are several situations in which the balance of risk and benefit of this strategy has much difficulties. Indeed, the risk of bleeding due to anticoagulant therapy is also critical $[3,4]$. Therefore, it is important to evaluate the risk-benefit of anticoagulant therapy.

Cerebral amyloid angiopathy (CAA) is caused by the accumulation of $\beta$-amyloid (amyloid $\beta 40$ proteins) in the cerebral arteries [5]. CAA is associated with intracerebral hemorrhage and the risk of recurrent bleeding is increased [6,7]. In addition, the risk of bleeding is presumed to be significantly enhanced in the treatment of anticoagulant therapy. On the other hand, direct oral anticoagulants (DOAC), such as edoxaban, have been shown to have a lower risk of cerebral bleeding as compared with warfarin in previous clinical trials [8]. However, the safety of DOAC for ischemic stroke in CAA patients with AF has not been obvious. We describe a CAA patient with ischemic stroke caused by AF in whom DOAC may be alternative to warfarin for the prevention of ischemic stroke without cerebral bleeding event.

\section{Case Presentation}

A 75-year-old man admitted to our outpatient clinic with an irregular pulse was diagnosed with AF by electrocardiogram. He had hypertension, diabetes, and previous stroke and scored 5 on the CHA2DS2-VASc (congestive heart failure, hypertension, age $\geqslant 75$ years, diabetes mellitus, stroke or transient ischemic attack, vascular disease, age 65 to 74 years, sex category) assessment of AF and stroke; treatment with vitamin $\mathrm{K}$ antagonist oral anticoagulant (warfarin) was initiated [9].

A few months later, he presented with a headache, and CAA was suspected after a brain MRI showed multiple hemorrhages in the cortical regions and cortical superficial siderosis with ischemic changes (Figure $1 A-1 C)$. We carefully adjusted and lowered the doses of warfarin while measuring the prothrombin time international normalized ratio (PT-INR). A follow-up MRI showed a recurrent cerebral hemorrhage due to CAA (Figure $1 D, 1 E$ ), and anticoagulant therapy was discontinued.

A few years after discontinuing warfarin, he was admitted with sudden-onset difficulties with left-hand movement, and a cerebral CT showed a cerebellar infarction (Figure 1F). A diffusion-weighted brain MRI showed other multiple new lesions of high signal intensity (Figure $1 G, 1 H$ ), but no thrombus was seen in a 


\section{Cureus}

transesophageal echocardiogram. To prevent a thrombotic stroke recurrence, however, we resumed anticoagulant therapy with a very small amount of edoxaban, gradually increasing the dose to $30 \mathrm{mg} / \mathrm{day}$ and monitoring the value of the D-dimer. There was one minor event of cerebral infarction before the dose increase (Figure 1I), but no events of cerebral hemorrhage and infarction have occurred in the several years since the edoxaban was initiated (Figure 1$)$ ).

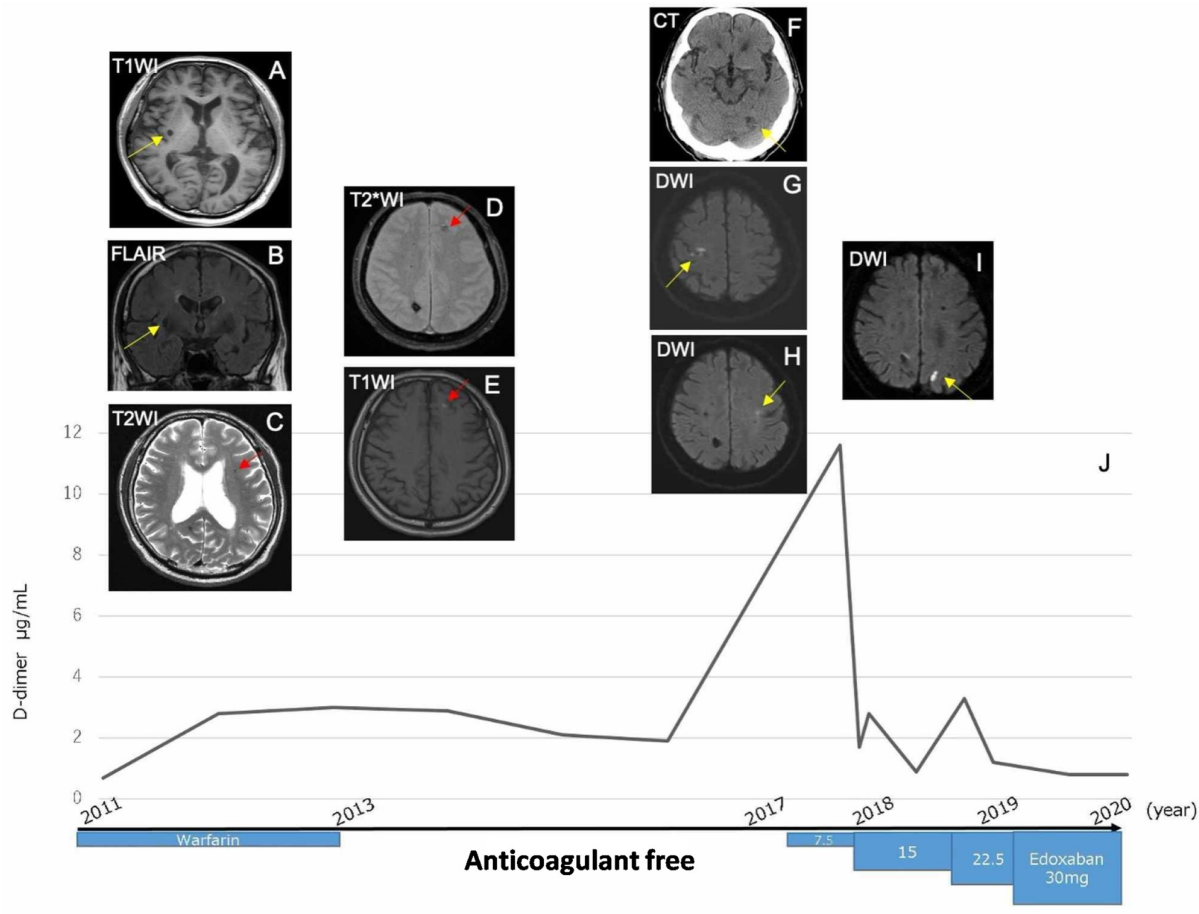

\section{FIGURE 1: Summary of the progress of this case}

A: T1-weighted image (T1WI) sequence showing a hypodensity lesion in the right putamen.

B: Axial fluid-attenuated inversion recovery (FLAIR) sequences showing an isodensity with $7 \mathrm{~mm}$ at the same lesion with $\mathrm{A}$, which suggested old cerebral bleeding.

C: T2WI sequence showing hemosiderosis in the left frontal lobe.

D: $2^{*}$ WI sequence showing a low-intensity lesion in the left frontal lobe.

E: T1WI sequence showing high intensities in the same lesion as D, in the left frontal lobe, which suggests subacute cerebral hemorrhage.

F: Cerebral CT showing left cerebellar infarction.

G, H: Diffusion-weighted image (DWI) sequence showing multiple deep and cortical infarct lesions, including right central groove lesion.

I: DWI sequence showing recurrence of cerebral infarction in other sites.

$\mathrm{J}$ : Time course of the level of D-dimer and medical treatment about anticoagulation.

\section{Discussion}

We describe a CAA patient with ischemic stroke caused by AF, which increases in prevalence with age and is the primary cause of cerebral infarction [10]. Anticoagulant therapy reduces the risk of cerebrovascular infarction in AF, but the benefits and risks of this strategy should be weighed in cases of hemorrhagic diathesis. Patients with CAA, which results from vascular injury by the accumulation of $\beta$-amyloid in the cerebral arteries, have a high risk of repeated intracerebral hemorrhage [11].

The mechanism of bleeding in CAA had not been concisely understood. Gurol et al. demonstrated the link between vascular amyloid burden and risk of bleeding [12]. In addition, the deposition of $\beta$-amyloid in the cerebrovascular export pathway resulted in further damage to blood vessels and aggravated CAA [13].

However, the risk of bleeding could not be predicted; therefore, there had been no helpful information about the decision about anticoagulant therapy, including warfarin [14]. There were some complex mechanisms 
relating oxidative stress underlying the mechanism of the progression of CAA. Han et al. demonstrated reactive oxygen species (ROS) and, in particular, nicotinamide adenine dinucleotide phosphate (NADPH) oxidase-derived ROS are a key mediator of CAA-induced vascular dysfunction and anti-ROS therapy attenuates CAA-related microhemorrhage [15]. Indeed, there were some reports about the anti-ROS effect of edoxaban. Narita et al. reported that edoxaban exerted antioxidant effects through factor Xa inhibition and direct radical-scavenging activity using human proximal tubular cells [16].

Although our patient had adverse bleeding events with warfarin several years earlier, he was successfully treated with edoxaban $[17,18]$. Rates of subdural and subarachnoid hemorrhage seem to be lower with DOAC than with warfarin [19]. Additionally, reactive oxygen stress is thought to play a role in CAA. The superiority of edoxaban in CAA might be due to its anti-ROS effects, which should be further explored in a large clinical studies [20].

\section{Conclusions}

We report the efficacy and safety of DOAC for the CAA with AF. Based on the experience of this case, early anticoagulant therapy with DOAC may be more appropriate than warfarin in coexistence case of CAA and AF.

\section{Additional Information \\ Disclosures}

Human subjects: Consent was obtained by all participants in this study. The University of Tokyo Institutional Review Board issued approval 2650. Conflicts of interest: In compliance with the ICMJE uniform disclosure form, all authors declare the following: Payment/services info: All authors have declared that no financial support was received from any organization for the submitted work. Financial relationships: All authors have declared that they have no financial relationships at present or within the previous three years with any organizations that might have an interest in the submitted work. Other relationships: EA belongs to the Department of Therapeutic Strategy for Heart Failure, Graduate School of Medicine, University of Tokyo, which is endowed by Actelion Pharmaceuticals Japan Ltd., Otsuka Pharmaceutical, NIPRO CORPORATION, Terumo Corp., Senko Medical Instrument Mfg., Century Medical Inc., Kinetic Concepts Inc., and St. Jude Medical.

\section{References}

1. Lip GYH, Collet JP, de Caterina R, et al.; ESC Scientific Document Group: Antithrombotic therapy in atrial fibrillation associated with valvular heart disease: a joint consensus document from the European Heart Rhythm Association (EHRA) and European Society of Cardiology Working Group on Thrombosis, endorsed by the ESC Working Group on Valvular Heart Disease, Cardiac Arrhythmia Society of Southern Africa (CASSA), Heart Rhythm Society (HRS), Asia Pacific Heart Rhythm Society (APHRS), South African Heart (SA Heart) Association and Sociedad Latinoamericana de Estimulación Cardíaca y Electrofisiología (SOLEACE). Europace. 2017, 19:1757-1758. 10.1093/europace/eux240

2. January CT, Wann LS, Calkins H, et al.: 2019 AHA/ACC/HRS focused update of the 2014 AHA/ACC/HRS guideline for the management of patients with atrial fibrillation: a report of the American College of Cardiology/American Heart Association Task Force on Clinical Practice Guidelines and the Heart Rhythm Society. J Am Coll Cardiol. 2019, 74:104-132. 10.1016/j.jacc.2019.01.011

3. Hart RG, Tonarelli SB, Pearce LA: Avoiding central nervous system bleeding during antithrombotic therapy: recent data and ideas. Stroke. 2005, 36:1588-1593. 10.1161/01.STR.0000170642.39876.f2

4. Toyoda K, Yasaka M, Iwade K, et al.; Bleeding with Antithrombotic Therapy (BAT) Study Group: Dual antithrombotic therapy increases severe bleeding events in patients with stroke and cardiovascular disease: a prospective, multicenter, observational study. Stroke. 2008, 39:1740-1745. 10.1161/STROKEAHA.107.504993

5. Attems J, Jellinger K, Thal DR, Van Nostrand W: Review: sporadic cerebral amyloid angiopathy. Neuropathol Appl Neurobiol. 2011, 37:75-93.

6. Viswanathan A, Greenberg SM: Cerebral amyloid angiopathy in the elderly. Ann Neurol. 2011, 70:871-880. 10.1002/ana.22516

7. Hemphill JC III, Greenberg SM, Anderson CS, et al.: Guidelines for the management of spontaneous intracerebral hemorrhage: a guideline for healthcare professionals from the American Heart Association/American Stroke Association. Stroke. 2015, 46:2032-2060. 10.1161/STR.0000000000000069

8. Granger CB, Alexander JH, McMurray JJ, et al.: Apixaban versus warfarin in patients with atrial fibrillation N Engl J Med. 2011, 365:981-992. 10.1056/NEJMoa1107039

9. Roldán V, Marín F, Manzano-Fernández S, et al.: The HAS-BLED score has better prediction accuracy for major bleeding than CHADS2 or CHA2DS2- VASc scores in anticoagulated patients with atrial fibrillation. J Am Coll Cardiol. 2013, 62:2199-2204. 10.1016/j.jacc.2013.08.1623

10. DeSimone CV, Graff-Radford J, El-Harasis MA, Rabinstein AA, Asirvatham SJ, Holmes DR Jr: Cerebral amyloid angiopathy diagnosis, clinical implications, and management strategies in atrial fibrillation. J Am Coll Cardiol. 2017, 70:1173-1182. 10.1016/j.jacc.2017.07.724

11. Keable A, Fenna K, Yuen HM, et al.: Deposition of amyloid b in the walls of human leptomeningeal arteries in relation to perivascular drainage pathways in cerebral amyloid angiopathy. Biochim Biophys Acta. 2016, 1862:1037-1046. 10.1016/j.bbadis.2015.08.024

12. Gurol ME, Dierksen G, Betensky R, et al.: Predicting sites of new hemorrhage with amyloid imaging in 


\section{Cureus}

cerebral amyloid angiopathy. Neurology. 2012, 79:320-326. 10.1212/WNL.0b013e31826043a9

13. Qi XM, Ma JF: The role of amyloid beta clearance in cerebral amyloid angiopathy: more potential therapeutic targets. Transl Neurodegener. 2017, 6:22. 10.1186/s40035-017-0091-7

14. Eckman MH, Wong LK, Soo YO, Lam W, Yang SR, Greenberg SM, Rosand J: Patient-specific decision-making for warfarin therapy in nonvalvular atrial fibrillation: how will screening with genetics and imaging help?. Stroke. 2008, 39:3308-3315. 10.1161/STROKEAHA.108.523159

15. Han BH, Zhou ML, Johnson AW, et al.: Contribution of reactive oxygen species to cerebral amyloid angiopathy, vasomotor dysfunction, and microhemorrhage in aged Tg2576 mice. Proc Natl Acad Sci USA. 2015, 112:E881-E890. 10.1073/pnas.1414930112

16. Narita Y, Hamamura K, Kashiyama M, et al.: Edoxaban exerts antioxidant effects through FXa inhibition and direct radical-scavenging activity. Int J Mol Sci. 2019, 20:4140. 10.3390/ijms20174140

17. O'Donnell MJ, Eikelboom JW, Yusuf S, et al.: Effect of apixaban on brain infarction and microbleeds: AVERROES-MRI assessment study. Am Heart J. 2016, 178:145-150. 10.1016/j.ahj.2016.03.019

18. Cannistraro RJ, Meschia JF: The clinical dilemma of anticoagulation use in patients with cerebral amyloid angiopathy and atrial fibrillation. Curr Cardiol Rep. 2018, 20:106. 10.1007/s11886-018-1052-1

19. Hart RG, Diener HC, Yang S, et al.: Intracranial hemorrhage in atrial fibrillation patients during anticoagulation with warfarin or dabigatran: the RE-LY trial. Stroke. 2012, 43:1511-1517. 10.1161/STROKEAHA.112.650614

20. Pfeifer M, Boncristiano S, Bondolfi L, et al.: Cerebral hemorrhage after passive anti-Abeta immunotherapy . Science. 2002, 298:1379. 10.1126/science.1078259 